\title{
Erratum to: Error Rates, Decisive Outcomes and Publication Bias with Several Inferential Methods
}

\author{
Will G. Hopkins ${ }^{1}$ - Alan M. Batterham ${ }^{2}$
}

Published online: 8 April 2016

(c) Springer International Publishing Switzerland 2016

\section{Erratum to: Sports Med \\ DOI 10.1007/s40279-016-0517-x}

Section 1, paragraph 3, lines 3-8: The following sentence, which previously read:

"An inference that the population value is substantial, clinically important, real or otherwise non-trivial—when, in reality, it is trivial-represents a false-positive or socalled type I error, whereas a false negative or type II error occurs when a trivial true value is inferred to be nontrivial”. should read:

"An inference that the population value is substantial, clinically important, real or otherwise non-trivial-when, in reality, it is trivial-represents a false-positive or so-called type I error, whereas a false negative or type II error occurs when a non-trivial true value is inferred to be trivial".

The online version of the original article can be found under doi:10.1007/s40279-016-0517-x.

\footnotetext{
Will G. Hopkins

will@clear.net.nz

1 Institute of Sport Exercise and Active Living, Victoria

University, Melbourne, VIC, Australia

2 Health and Social Care Institute, Teesside University, Middlesbrough, UK
} 